\title{
Anastrozole/Palbociclib Regimen
}

National Cancer Institute

\section{Source}

National Cancer Institute. Anastrozole/Palbociclib Regimen. NCI Thesaurus. Code C156367.

A chemotherapy regimen consisting of anastrozole and palbociclib that is used in the treatment of estrogen receptor (ER)-positive advanced breast cancer. 